\title{
Designed to linger
}

DOI:

$10.1038 /$ nrd2430
Growth hormone $(\mathrm{GH})$ is a key cytokine for stimulating body growth and recombinant human $\mathrm{GH}$ is commonly used to treat short stature. However, owing to its short half-life, current therapeutic regimens require daily injections. In an attempt to

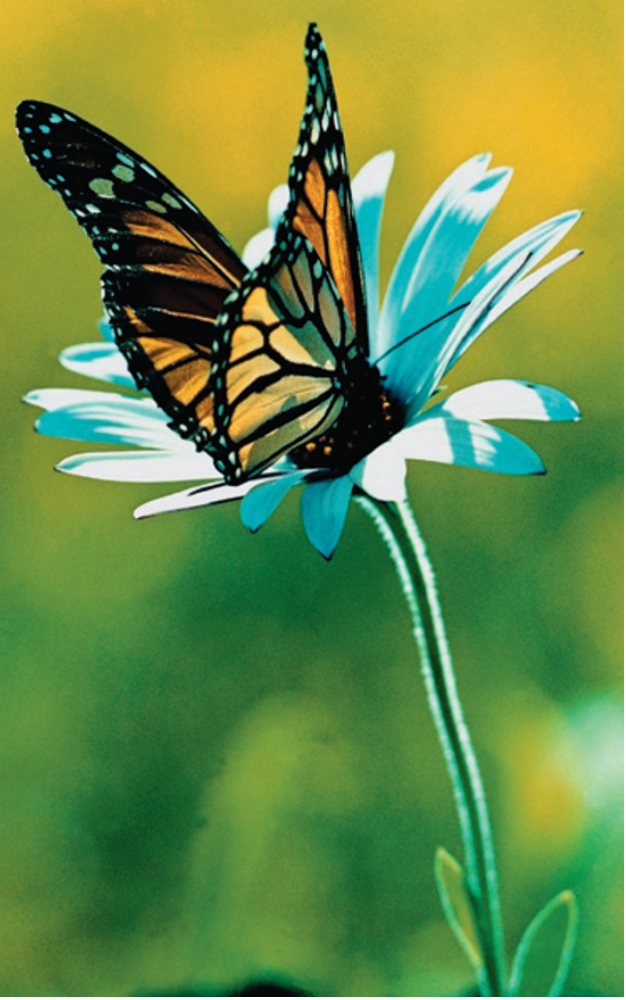

design a GH antagonist, Wilkinson and colleagues have discovered a potent and long-lasting GH agonist, which holds promise for future GH replacement therapies, and suggest a general strategy for the design of more effective cytokine receptor agonists.

Excess GH is the main cause of pituitary tumours, gigantism and acromegaly. Previous studies have shown that GH receptor variants in which the extracellular domain is truncated function as dominant negative inhibitors of GH signalling. The authors predicted that a $\mathrm{GH}$-receptor fusion protein would change the conformation of the $\mathrm{GH}$ receptor and block GH signalling. They generated a recombinant gene by linking the $\mathrm{GH}$ gene to the $\mathrm{A}$ and $\mathrm{B}$ extracellular domains of the $\mathrm{GH}$ receptor through a flexible linker and purified the $75 \mathrm{kDa}$ protein product from Chinese hamster ovary cells.

To their surprise, they found that this ligand-receptor fusion protein was able to induce a maximal response in a $\mathrm{GH}$-specific reporter assay. Interestingly, when it was injected either subcutaneously or intravenously into rats, traces of the ligand-receptor fusion protein were detectable after 8 days, indicating a remarkable delay in clearance compared with recombinant $\mathrm{GH}$, which was undetectable after only 6 hours.

To assess the therapeutic potential of this fusion protein, the authors compared the effects of administering the same $\mathrm{GH}$ dose over 10 days, as $\mathrm{GH}$ or GH-receptor fusion protein, using different injection regimens. A single injection of GH-receptor induced a greater weight gain than a single GH injection, and similar weight gain to daily $\mathrm{GH}$ injections. GH only seemed to promote weight gain 24 hours after injection, whereas the effects of the fusion protein were still observed after 10 days. Similarly, the levels of insulin-like growth factor, a biomarker of GH activity, were significantly higher after a single injection of GH-receptor fusion protein than after daily injections of GH.

The authors propose that the long-lasting effects of the ligandreceptor fusion protein are due to the formation of reciprocal headto-tail dimers, and that this conformation prevents proteolysis while enabling the GH moiety to activate $\mathrm{GH}$ receptors. Fusing cytokine hormones to the extracellular domain of their receptors might therefore be a cheap and simple way of generating potent cytokine receptor agonists with a long duration of action for therapy of a wide range of disorders.

Monica Hoyos Flight

ORIGINAL RESEARCH PAPER Wilkinson, I. A. et al. A ligand-receptor fusion of growth hormone forms a dimer and is a potent longacting agonist. Nature Med. 13, 1108-1113 (2007) 